\title{
Variation on agro-morphological traits in Nepalese foxtail millet (Setaria italica (L) P Beauv)
}

\author{
RB Amgai, S Pantha, TB Chhetri, SK Budhathoki, SP Khatiwada, and A Mudwari*
}

\begin{abstract}
Foxtail millet (Setaria italica (L) P Beauv) falls on the category of underutilized crops in Nepal and mainly cultivated in Karnali region of the country. It is hardy crop and considered as one of the potential crops for future food security with respect to climate change. Five accessions of Nepalese foxtail millet were purposefully selected for evaluation of the agromorphological characteristics. Foxtail landraces from Dolpa, Mugu, Bajura, Bajhang and Lamjung districts of Nepal were evaluated at Khumaltar, Lalitpur, Nepal during 2010. The plot size was $1 \mathrm{~m}^{2}$ and there were five samples. Days to heading and days to maturity varied from 33-56 and 59 to 87 days after germination respectively. Similarly, flag leaf length/breadth ratio, flag leaf sheath length, ligule length, peduncle length, peduncle exertion and plant height varied from 3.84-10.90, 5.47-9.84 cm, 0.1-0.2 mm, 10-22.57 cm, $2.7-13.58 \mathrm{~cm}$ and $41.67-120 \mathrm{~cm}$, respectively. Fruit and apiculus color varied from straw to black. All accessions were actively growing with very slight lodging. Similarly, the thousand grain weight varies from $1.064 \mathrm{~g}$ to $2.172 \mathrm{~g}$. This variation is useful in foxtail millet breeding program. Similarly, the significant correlation between thousand kernel weight and total basal tiller $(\mathrm{r}=-0.975)$ showed that foxtail millet lines with low tillering ability is better for yield enhancement.
\end{abstract}

Key words: Fox-tail millet, agro-morphological, land races, germplasm

\section{Introduction}

Foxtail millet (Setaria italica (L) P Beauv) has been in cultivation for about 8,000 years in China. It was cultivated as an important food across southern Europe until the early 20th century (Austin, 2006). It is small grain millet mostly cultivated in marginal and nonirrigated condition of mid and high hill areas of Nepal especially in Karnali region of Nepal. Nepal has high genetic diversity in foxtail millet (HMGN/MFSC, 2002). However, very little research has been conduction on this crop. Therefore, its status is still unexplored and under utilized. It has the great potentiality for food security. Crop hardiness has higher scope for combating effects of climate change by this crop. Therefore, major attention has to be given for exploration, evaluation, and utilization of the foxtail millet for its commercial cultivation. Varietal development through landrace enhancement is one of the best options.

*Agriculture Botany Division, Khumaltar, NARC. reshamamgain@yahoo.com 
Nepalese foxtail millet landraces can be grouped into tropical group based on Pro2f allele of prolamin but Pro2b allele was uncommon in them (Nakayama et al., 1999). Similarly, Kawase and Sakamoto (1984) also found variation in esterase in Nepalese foxtail millets. Positive type for phenol coloration was primarily found in accessions from Taiwan, Philippines, Nepal, and India (Kawase and Sakamoto 1982).

\section{Material and methods}

Five accessions of Nepalese foxtail millet were purposefully selected for evaluation of the agro-morphological characteristics (Table 1). Foxtail landraces from Dolpa, Mugu, Bajura, Bajhang and Lamjung were evaluated during 2010 at Khumaltar, Lalitpur in $1 \mathrm{~m}^{2}$ plots with two replications. Plants are sown continuously with $20 \mathrm{~cm}$ row spacing. Days to $50 \%$ plants showing heading and 50\% plants showing maturity after germination were recorded. Similarly, other characters are evaluated based on foxtail millet descriptor (IBPGR, 1985).

Table 1. List of the foxtail millet germplasm used for agro-morphology study at Khumaltar during 2010

\begin{tabular}{clc}
\hline S. No. & Collection district & Altitude of collected site \\
\hline 1 & Lamjung & 1402 \\
2 & Bajura & 1829 \\
3 & Bajhang & 1829 \\
4 & Mugu & 2575 \\
5 & Dolpa & 2280 \\
\hline
\end{tabular}

Data from five individual primary tillers were measured. MS Excel, Minitab and SPSS for windows were used for data analysis

\section{Results}

A lot of variation was observed among Nepalese foxtail millet landraces for flag leaf length/breadth ratio, flag leaf sheath length, ligule length, no. of branches/plant, no. of basal tillers/plant, no. of effective tillers/plant, peduncle length, peduncle exertion, plant height and thousand grain weight (Table 2). Similar type of variation was also observed (Reddy et al; 2006) while studying 21 accessions of Nepalese foxtail millet at ICRISAT, India. Similarly, growth habit, blade pubescence, lodging, senescence, fruit color and apiculus color also showed great variation among accessions. All accessions had green leaf and without pigmentation on plant parts (Table 3). Thousand grains weight was found highest in Bajhang and lowest in Bajura. Lamjung took longest days to flowering and maturity, however, Bajura had shortest days to flowering and maturity after germination. 
Agronomy Journal of Nepal (Agron JN) Vol. 2: 2011

Table 2. Mean of different characters in foxtail millet germplasm observed at Khumaltar during 2010

\begin{tabular}{lccccccccccc}
\hline $\begin{array}{c}\text { Accession } \\
\text { Name }\end{array}$ & $\begin{array}{c}\text { Flag leaf length/ } \\
\text { breadth ratio }\end{array}$ & $\begin{array}{c}\text { Flag leaf } \\
\text { sheath } \\
\text { length, cm }\end{array}$ & $\begin{array}{c}\text { Ligule } \\
\text { Length, } \\
\text { mm }\end{array}$ & $\begin{array}{c}\text { No. of } \\
\text { branches }\end{array}$ & $\begin{array}{c}\text { No. of } \\
\text { basal } \\
\text { tillers }\end{array}$ & $\begin{array}{c}\text { No. of } \\
\text { effective } \\
\text { tillers }\end{array}$ & $\begin{array}{c}\text { Peduncle } \\
\text { length, cm }\end{array}$ & $\begin{array}{c}\text { Peduncle } \\
\text { exertion, } \\
\text { cm }\end{array}$ & $\begin{array}{c}\text { Plant } \\
\text { Height, cm }\end{array}$ & $\begin{array}{c}\text { 1000 grain } \\
\text { weight, gm }\end{array}$ \\
\hline Lamjung & 3.83 & 8.63 & 0.20 & 0.17 & 1.67 & 0.83 & 10.00 & 2.70 & 120.00 & 1.11 \\
Bajura & 8.29 & 6.93 & 0.10 & 1.00 & 1.67 & 1.67 & 17.67 & 9.67 & 41.67 & 1.06 & 70 \\
Bajhang & 10.91 & 9.83 & 0.10 & 0.00 & 1.17 & 1.00 & 22.57 & 13.58 & 70.00 & 2.17 \\
Mugu & 8.29 & 8.25 & 0.10 & 0.00 & 1.67 & 1.67 & 14.25 & 7.00 & 46.50 & 1.34 \\
Dolpa & 8.96 & 5.47 & 0.12 & 0.00 & 1.17 & 1.00 & 15.50 & 9.00 & 52.50 & 2.03 \\
\hline
\end{tabular}

Table 3. Variation in different characters observed among Nepalese foxtail millet landraces at Khumaltar during 2010

\begin{tabular}{lcccccccccccc}
\hline $\begin{array}{c}\text { Accession } \\
\text { name }\end{array}$ & $\begin{array}{c}\text { Mean } \\
\text { days to } \\
\text { heading }\end{array}$ & $\begin{array}{c}\text { Mean } \\
\text { days to } \\
\text { flowering }\end{array}$ & $\begin{array}{c}\text { Mean } \\
\text { days to } \\
\text { maturity }\end{array}$ & $\begin{array}{c}\text { Growth } \\
\text { Habit }\end{array}$ & $\begin{array}{c}\text { Plant } \\
\text { pigmenta } \\
\text { tion }\end{array}$ & $\begin{array}{c}\text { Leaf } \\
\text { color }\end{array}$ & $\begin{array}{c}\text { Blade } \\
\text { pubescen } \\
\text { ce }\end{array}$ & Lodging & Senescence & Fruit color & $\begin{array}{c}\text { Apiculus } \\
\text { color }\end{array}$ \\
\hline Lamjung & 56 & 62 & 87 & 1 & 0 & Green & 5 & 1 & 9 & Black & Black \\
Bajura & 33 & 38 & 59 & 1 & 0 & Green & 1 & 1 & 1 & Brown & Brown \\
Bajhang & 49 & 52 & 78 & 2 & 0 & Green & 1 & 5 & 1 & Straw & Straw \\
Mugu & 49 & 52 & 63 & 1 & 0 & Green & 1 & 1 & 1 & Straw & Straw \\
Dolpa & 48 & 50 & 69 & 1 & 0 & Green & 1 & 1 & 9 & Black spots & Black \\
\hline
\end{tabular}


Foxtail millet landrace collected from Lumjung district fell on the distinct group than other landraces. Similarly, landraces from Bajura and Mugu grouped on the same group in $99 \%$ of similarity (Figure 1).



Plant height had significant positive correlation with days to maturity $(\mathrm{r}=0.954)$ and ligule length $(\mathrm{r}=0.923)$. Positive correlation between plant height and days to maturity was also observed by Nirmalakumari and Vetriventhan (2010). Peduncle exertion was highly correlated with peduncle length $(\mathrm{r}=0.987)$. Similarly, thousand grains weight was negatively correlated with total basal tiller $(\mathrm{r}=-0.975)$. Flag leaf length/breadth ration was positively correlated with peduncle length $(\mathrm{r}=0.911)$ and peduncle exertion $(\mathrm{r}=0.954)$. However, days to maturity had negative correlation with total effective tiller $(\mathrm{r}=-0.89)$ (Table 3).

\section{Discussion}

Higher level of variability can be found in Nepalese foxtail millet. Therefore, Nakayama et al; (1999) concluded that Nepal could be one of the centers of diversity for foxtail millet. Landrace from Lamjung district showed longer growing duration. It fell also in distinct group (Figure 1). This landrace was collected from low altitude area. These particular characteristics may be contributed for its distinct grouping. Negative correlation between days to maturity and total effective tiller and positive correlation between days to maturity and plant height (Table 4) showed that there was very low synchronization between the maturity of primary tiller and secondary tillers. Therefore, low tiller bearing landraces should be selected for commercial cultivation. Ochiai (1996) also considered high tillering accessions as recently originated from their wild progenitors. Similarly, thousand grains weight was found reduced when there was higher numbers of basal tillers as shown by their negative correlation. However, the positive relationship between total basal tillers and total effective tillers (Nirmalakurmari and Vetriventhan, 2010) should give the positive correlation with grain yield as observed by Channappagoudar et al. (2008). Our case may be due to unsynchronized maturity imposed by high tillering. 
Agronomy Journal of Nepal (Agron JN) Vol. 2: 2011

Table 4. Correlation between different traits observed in Nepalese foxtail millet at Khumaltar observed during 2010 (n=5)

\begin{tabular}{|c|c|c|c|c|c|c|c|c|c|c|c|}
\hline & $\begin{array}{l}\text { Days to } \\
\text { heading }\end{array}$ & $\begin{array}{c}\text { Days to } \\
\text { maturity }\end{array}$ & $\begin{array}{c}\text { Flag leaf } \\
\text { length/ } \\
\text { breadth } \\
\text { ratio }\end{array}$ & $\begin{array}{l}\text { Flag leaf } \\
\text { sheath } \\
\text { length, cm }\end{array}$ & $\begin{array}{c}\text { Ligule } \\
\text { length, } \\
\text { mm }\end{array}$ & $\begin{array}{c}\text { No. of } \\
\text { branches }\end{array}$ & $\begin{array}{l}\text { Total basal } \\
\text { tiller }\end{array}$ & $\begin{array}{c}\text { Total } \\
\text { effective } \\
\text { tiller }\end{array}$ & $\begin{array}{l}\text { Peduncle } \\
\text { length, cm }\end{array}$ & $\begin{array}{c}\text { Peduncle } \\
\text { exertion, } \\
\text { cm }\end{array}$ & $\begin{array}{c}\text { Plant } \\
\text { height, } \mathrm{cm}\end{array}$ \\
\hline Days to maturity & 0.801 & & & & & & & & & & \\
\hline $\begin{array}{l}\text { Flag leaf length/ } \\
\text { breadth ratio }\end{array}$ & -0.39 & -0.459 & & & & & & & & & \\
\hline $\begin{array}{l}\text { Flag leaf sheath } \\
\text { length, cm }\end{array}$ & 0.394 & 0.512 & 0.005 & & & & & & & & \\
\hline Ligule length, $\mathrm{mm}$ & 0.627 & 0.779 & -0.899 & 0.116 & & & & & & & \\
\hline No. of branches & -0.849 & -0.482 & -0.107 & -0.26 & -0.147 & & & & & & \\
\hline Total basal tiller & -0.162 & -0.185 & -0.661 & 0.094 & 0.295 & 0.492 & & & & & \\
\hline Total effective tiller & -0.7 & $-0.89 *$ & 0.246 & -0.166 & -0.643 & 0.523 & 0.529 & & & & \\
\hline Peduncle length, $\mathrm{cm}$ & -0.437 & -0.262 & $0.911^{*}$ & 0.236 & -0.761 & 0.081 & -0.6 & 0.13 & & & \\
\hline Peduncle exertion, $\mathrm{cm}$ & -0.453 & -0.333 & $0.954 *$ & 0.1 & -0.807 & 0.045 & -0.666 & 0.139 & $0.987 * *$ & & \\
\hline Plant height, $\mathrm{cm}$ & 0.723 & $0.941 *$ & -0.721 & 0.452 & $0.923^{*}$ & -0.276 & 0.139 & -0.749 & -0.504 & -0.588 & \\
\hline Thousand grain weight & 0.238 & 0.164 & 0.719 & 0.031 & -0.369 & -0.612 & $-0.975^{*}$ & -0.456 & 0.624 & 0.683 & -0.176 \\
\hline
\end{tabular}

Note: $* *=$ significant at $(\mathrm{P}<0.01)$ level of significance and $*=$ significant at $(\mathrm{P}<0.05)$ level of significance 


\section{Conclusion}

Nepalese foxtail millet landraces showed higher level of variability in many traits. This variation can be used for further breeding program. However, landraces producing low tillers should be selected for yield improvement. Similarly, maturity synchronization between primary and secondary tillers should be considered.

\section{References}

Austin, DF. 2006. Fox-tail Millets (Setaria: Poaceae)-abandoned food in two hemispheres. Economic Botany 60(2): 143-158.

Channappagoudar BB, SM Hiremath, NR Biradar, RV Koti and TD Bharamagoudar. 2008. Physiological Basis of Yield Variation in Foxtail Millet. Karnataka Journal of Agricultural Science 20(3): 481-486.

HMGN/MFSC (His Majesty's Government of Nepal/Ministry of Forests and Soil Conservation), Nepal. 2002. Nepal Biodiversity Strategy. Ministry of Forests and Soil Conservation, HMG, Nepal. $117 \mathrm{p}$.

IBPGR (International Board for Plant Genetic Resources). 1985. Descriptors for Setaria italica and S pumila. International Board for Plant Genetic Resources. Rome. 18p.

Kawase, M and S Sakamoto. 1982. Geographical distribution and genetic analysis of phenol color reaction in foxtail millet, Setaria italica (L.) P. Beauv. Theoretical Applied Genetics 63:117119 .

Kawase, M and S Sakamoto. 1984. Variation, geographical distribution and genetical analysis of esterase isozymes in foxtail millet, Setaria italica (L.) P. Beauv. Theoretical and Applied Genetics 67 (6): 529-533.

Nakayama, H, H Namai and K Okuno. 1999. Geographical variation of the alleles at the two prolamin loci, Pro1 and Pro2, in foxtail millet, Setaria italica (L.) P. Beauv. Genes Genet. Syst. 74: 293-297.

Nirmalakumari, A and M Vetriventhan. 2010. Characterization of foxtail millet germplasm collections for yield contributing traits Electronic Journal of Plant Breeding 1(2): 140-147.

Ochiai. 1996. Variation in tillering and geographical distribution in foxtail millet (Setaria italica P. Beauv). Breeding Science 46:143-148.

Reddy, VG, HD Upadhyaya and CLL Gowda. 2006. Characterization of World's Foxtail Millet Germplasm Collections for Morphological Traits. SAT eJournal (ejournal.icrisat.org) 2 (1): 107-109. 